\title{
Structure, dielectric and ferroelectric properties of perovskite ceramics
}

Ekaterina Politova ${ }^{1}$, Natalia Golubko ${ }^{1}$, Galina Kaleva ${ }^{1}$, Aleksander Mosunov ${ }^{1}$, Natalia Sadovskaya ${ }^{1}$, Sergey Stefanovich ${ }^{1}$, Prasanta Panda ${ }^{2}$

${ }^{1}$ L.Ya. Karpov Insitute of Physical Chemistry, Moscow, Russian Federation, ${ }^{2}$ National Aerospace Laboratories, Kodihalli, Bangalore560017 , Bangalore, India

E-mail: politova@cc.nifhi.ac.ru

Due to increasing concern on the environment safety, new lead-free piezoelectric materials are being intensively studied in order to replace widely used $\mathrm{Pb}$-based ones. Oxides with perovskite structure on the base of sodium-potassium niobate $(\mathrm{K} 0.5 \mathrm{Na0} .5) \mathrm{NbO} 3(\mathrm{KNN})$ and bismuth-sodium titanate (Na0.5Bi0.5)TiO3 (NBT) are being considered among the most promising ones. In addition to addressing environmental challenges, development of new highly efficient oxide materials is of great scientific interest to the fields of physics, chemistry and materials science. As basic approach for creating new piezoelectric materials, modification of compositions close to Morphotropic Phase Boundaries (MPB) which divide concentration regions with different crystal structures is mainly used.

In this work, compositions close to the MPB in the (K0.5Na0.5)NbO3 (KNN) and (Na1/2Bi1/2)TiO3 - BaTiO3 (NBT-BT) systems have been studied. Effects of modification of compositions with acceptor dopants (manganese, iron and nickel cations in the B-sites of perovskite lattice) and influence of over stoichiometric additives with low melting temperatures (Bi2O3, KCl, LiF and V2O5) on structure, dielectric and ferroelectric properties of ceramics were studied.

Ceramic samples were prepared by the two-step solid-state reaction method at temperatures $800-12000 \mathrm{C}$. $\mathrm{KCl}$ and $\mathrm{LiF}$ were added to the NBT-BT composition in amounts up to $15 \mathrm{~mol}$ \%. Bi2O3, V2O5, Mn2O3, Ni2O3 and Fe2O3 were added to the KNN- and NBT-based compositions in amounts of $5-15 \mathrm{~mol}$. \%.

The samples were characterized using the X-ray Diffraction, Differential Thermal Analysis (DTA) Scanning Electron Microscopy (SEM), Second Harmonic Generation (SHG), and Dielectric Spectroscopy methods.

In the BNT-BT compositions the unit cell volume increased in compositions doped by $\mathrm{KCl}, \mathrm{Bi} 2 \mathrm{O} 3$, did not changed in compositions doped by LiF and decreased in compositions doped by V2O5 additives. Mn3+ and Fe3+ cations stimulated increase in the unit cell volume of BNT-based compounds, practically did not influence the unit cell volume of KNN-based ones, while influence of $\mathrm{Ni3}+$ cations was opposite.

Phase transitions were marked by steps near 350-400 $\mathrm{K}$ and by peaks at $\sim 550 \mathrm{~K}$ in dielectric permittivity versus temperature curves of NBT-based compositions and by peaks at $\sim 700 \mathrm{~K}$ in these curves of KNN-based compositions. Increase in the spontaneous polarization value was proved for modified ceramics using the SHG method.

At the room temperature, non monotonous changes in the dielectric permittivity value ert were observed in modified by BNT-BT and KNN-based compositions.

Effects of dielectric relaxation related to the presence of oxygen vacancies in compositions containing B-site cations with mixed valence and with deficiency in the A-sites of the perovskite lattice were observed at high temperatures $>700 \mathrm{~K}$.

The results obtained confirmed prospects of new lead-free materials development by modification of KNN- and NBT-based compositions close to the MPB by aliovalent cation substitutions.

Acknowledgment

The work was supported by the Russian Fund for Basic Research (Project 16-53-48009).

Keywords: perovskite structure, aliovalent cation substitutions 